\section{Uso de medicamentos potencialmente inadequados entre idosos do Município de Viçosa, Minas Gerais, Brasil: um inquérito de base populacional}

\author{
Use of potentially inappropriate medications in \\ the elderly in Viçosa, Minas Gerais State, Brazil: \\ a population-based survey
}

\section{Uso de medicamentos potencialmente inadecuados entre ancianos de Viçosa, Minas Gerais, Brasil: un estudio basado en la población}

\begin{abstract}
The study evaluated the use of potentially inappropriate medications among elderly in Viçosa, Minas Gerais State, Brazil, according to the Beers 2012 and STOPP criteria, as well as factors associated with this use. This was a cross sectional study of 621 community-dwelling elderly using home interviews. The explanatory variables were gender, age, education, self-rated health, restriction of activities in the previous 15 days, functional capacity, history of hospital admission, number of self-reported diseases, and polypharmacy. Multivariate Poisson regression was used. There was a high prevalence of potentially inappropriate medications according to the Beers 2012 and STOPP criteria: 43.8\% (95\%CI: 37.8\%-47.8\%) and 44.8\% (95\%CI: 40.9\%-48.8\%), respectively. Female gender and polypharmacy remained independently associated with use of potentially inappropriate medications according to the Beers criteria. As for the STOPP criteria, the variables independently associated with use of potentially inappropriate medications were female gender, fair self-rated health, and polypharmacy. Efforts are needed to upgrade polypharmacy in Brazilian elders.
\end{abstract}

Drug Utilization; Polypharmacy; Health of the Elderly; Health Surveys
Gabriela Aires Martins 1

Francisco de Assis Acurcio 1

Sylvia do Carmo Castro Franceschini 2

Silvia Eloiza Priore 2

Andréia Queiroz Ribeiro ${ }^{2}$

\section{Resumo}

Avaliou-se o uso de medicamentos potencialmente inadequados entre idosos de Viçosa, Minas Gerais, Brasil, de acordo com os critérios de Beers 2012 e STOPP, bem como os fatores associados a esse uso. Estudo transversal com 621 idosos não institucionalizados, abordados por entrevista domiciliar. As variáveis explicativas foram sexo, idade, escolaridade, percepção da saúde, restrição de atividades nos últimos 15 dias, capacidade funcional, história de internação hospitalar, número de doenças autorreferidas e polifarmácia. Realizou-se análise de regressão de Poisson multivariada. Observou-se alta prevalência de uso de medicamentos potencialmente inadequados, 43,8\% (IC95\%: 37,8\%47,8\%) e 44,8\% (IC95\%: 40,9\%-48,8\%), segundo os critérios de Beers 2012 e STOPP respectivamente. Sexo feminino e polifarmácia se mantiveram independentemente associados ao uso de medicamentos potencialmente inadequados, de acordo com os critérios de Beers. Para o critério STOPP, as variáveis independentemente associadas ao uso de medicamentos potencialmente inadequados foram sexo feminino, percepção de saúde regular e polifarmácia. Esforços são necessários para se qualificar a prática da polifarmácia entre idosos.

Uso de Medicamentos; Polimedicação; Saúde do Idoso; Inquéritos Epidemiológicos 


\section{Introdução}

O fenômeno do envelhecimento populacional, especialmente nos países em desenvolvimento, delineia um cenário de desafios em diferentes setores da sociedade, de forma a garantir adequadas condições de vida a esse segmento etário 1. No Brasil, em 2012, o número de idosos atingiu mais de 23,5 milhões de pessoas, o que representa $12,6 \%$ da população ${ }^{2}$. Estimativas mais conservadoras indicam que o país se tornará o sexto no mundo em número de idosos já em 2020, com um contingente superior a 30 milhões de pessoas 3 . Esse envelhecimento tem como consequências aumento da prevalência de doenças crônicas não transmissíveis, de incapacidades, bem como aumento na demanda por serviços de saúde e de benefícios 4,5. A elevada prevalência de múltiplas doenças crônicas e sua coexistência, associadas a mudanças fisiológicas relacionadas ao envelhecimento, favorece a exposição da população idosa ao uso de múltiplos medicamentos e ao surgimento de problemas relacionados a esse uso 6,7,8.

Nesse contexto, estudos têm demonstrado que a polifarmácia reduz a adesão à terapêutica medicamentosa, aumenta a frequência e gravidade das reações adversas e interações medicamentosas, o risco de utilização de medicamentos potencialmente inadequados e, consequentemente, a morbimortalidade 9,10,11,12,13. Medicamentos potencialmente inadequados são aqueles que devem ser evitados em idosos, visto que o risco de eventos adversos supera o benefício e que existe uma alternativa terapêutica mais segura e eficaz para a condição clínica 14,15.

Logo, o aprimoramento da farmacoterapia em idosos prescinde da identificação da magnitude e dos determinantes do uso inadequado de medicamentos. Fatores como presença de múltiplas comorbidades, sexo feminino, idade avançada, automedicação e número de medicamentos utilizados demonstram estar associados com essa prática no cenário internacional 16 .

Vários critérios vêm sendo desenvolvidos e estudados mundialmente com o objetivo de avaliar o uso de medicamentos potencialmente inadequados e sua consequência entre idosos 14,17. A Lista de Beers são os critérios mais utilizados desde a sua criação, em 1991, e se encontra na quarta atualização 18. Tal lista engloba medicamentos ou classes de medicamentos divididos em três categorias: medicamentos potencialmente inadequados que devem ser evitados em idosos, medicamentos potencialmente inadequados que devem ser evitados em idosos portadores de determinadas doenças, as quais podem ser agravadas por tais medicamentos, e medi- camentos que devem ser usados com cuidado em idosos 18. No entanto, algumas limitações dessa lista são apresentadas por pesquisadores. Por exemplo, cerca de $50 \%$ dos medicamentos listados não estão presentes nos formulários de países europeus, a lista inclui medicamentos que não são completamente contraindicados em idosos, além de não contemplar interações entre medicamentos, apresentar duplicidades de classes terapêuticas e omitir medicamentos que deveriam ser utilizados 14,19 .

Diante dessas limitações, foram criados, em 2008, na Europa, os critérios Screening Tool of Older Person's Prescriptions/Screening Tool to Alert doctors to Right Treatment (STOPP/START). Tais critérios são baseados em evidências clínicas atualizadas e têm o intuito de auxiliar o prescritor, de forma rápida e fácil, a detectar, corrigir e prevenir, em idosos, a prescrição inadequada e a falta de prescrição de medicamentos indicados, levando em consideração a presença de múltiplas doenças. Cada critério STOPP apresenta uma justificativa concisa de por que se considera inadequada a prescrição para idosos, com base na avaliação das interações entre medicamentos e entre medicamento e enfermidades. Já os critérios START avaliam erros por omissões de prescrição que provavelmente beneficiaria os pacientes idosos 14 .

Por sua vez, no Brasil, dados sobre a prevalência de uso de medicamentos potencialmente inadequados em idosos não institucionalizados são limitados. Em pesquisas anteriores, essa prevalência variou de $21,7 \%$ a 59,2\% 20,21,22,23,24. Diante do exposto, o objetivo do presente estudo foi avaliar o uso de medicamentos potencialmente inadequados entre idosos do Município de Viçosa, Minas Gerais, segundo os critérios de Beers 2012 e os critérios STOPP, bem como identificar os fatores associados a este uso.

\section{Métodos}

Os dados analisados fazem parte do projeto de pesquisa intitulado Condições de Saúde, Nutrição e Uso de Medicamentos por Idosos do Município de Viçosa, MG: Um Inquérito de Base Populacional, desenvolvido pelo Departamento de Nutrição e Saúde, Universidade Federal de Viçosa. A população-alvo deste estudo é constituída pelos idosos da comunidade, com 60 ou mais anos, residentes no Município de Viçosa, incluindo as zonas urbana e rural.

Em 2007, a cidade contava com uma população de 7.034 indivíduos na faixa etária em questão, conforme apontam os resultados da contagem populacional daquele ano. Essa po- 
pulação foi recenseada durante a Campanha Nacional de Vacinação do Idoso, no período de abril a maio de 2008. Com o objetivo de identificar os não participantes da campanha de vacinação e complementar a base cadastral, procedeu-se à confrontação e junção desse banco de dados com outros disponíveis no município, a saber: o dos Servidores da Universidade Federal de Viçosa ativos e aposentados com 60 anos e mais de idade; os cadastros dos idosos do Programa Saúde da Família (PSF), do serviço de fisioterapia municipal, do centro de saúde da mulher, do serviço psicossocial, da unidade de atendimento, do HiperDia e da Policlínica. Após essa junção dos bancos, o registro de pessoas com 60 anos e mais totalizou 7.980, servindo de base para a obtenção da amostra. Idosos institucionalizados foram excluídos da população de referência.

O cálculo do tamanho amostral considerou um nível de 95\% de confiança, prevalências estimadas de $50 \%$ para diferentes desfechos estudados e erro tolerado de $4 \%$. Assim, a amostra seria de 558 idosos, à qual se acrescentaram $20 \%$ para cobrir possíveis perdas, totalizando 670 pessoas a serem estudadas. Ocorreram perdas de informação por recusa $(3,6 \%)$ e por motivos inevitáveis para a realização das entrevistas $(3,7 \%)$. Consideraram-se perdas inevitáveis as situações em que foram sorteados indivíduos que haviam falecido (1,3\%), endereços não localizados $(1,2 \%)$ e aqueles que se mudaram para residências de difícil localização ou para outros municípios $(1,2 \%)$. Assim, foram efetivamente estudados 621 idosos.

A seleção dos participantes foi realizada valendo-se do cadastro, por amostragem aleatória simples.

Um estudo-piloto foi realizado previamente à coleta de dados com os objetivos de verificar a adequação da dinâmica de recrutamento; testar o instrumento de coleta de dados; avaliar o tempo médio de duração das entrevistas e confirmar a viabilidade da investigação.

As entrevistas ocorreram de março a dezembro de 2009 no nível domiciliar. Os questionários foram aplicados diretamente ao idoso, por duplas de entrevistadores previamente treinados, $\mathrm{e}$ caso ele apresentasse dificuldade, o respondente próximo o auxiliava. Os instrumentos foram revisados e codificados sob a supervisão de um coordenador de campo; após revisão, foram liberados para digitação.

As informações para o presente trabalho, relacionadas a características sociais e econômicas, condições de saúde, uso de serviços de saúde e de medicamentos nos 15 dias anteriores à realização da entrevista, foram obtidas mediante um questionário semiestruturado. Para evitar viés de memória, os dados sobre uso de medicamentos foram obtidos com período recordatório de 15 dias. A maioria dos medicamentos teve seu uso comprovado por bulas, embalagens ou prescrições apresentadas pelos participantes.

As unidades de análise foram os medicamentos e os indivíduos. Os medicamentos foram classificados de acordo com o Anatomical Therapeutic Chemical (ATC) Classification System (World Health Organization. http://www.whocc. no/atc_ddd_index/, acessado em 05/Abr/2014) e agrupados conforme o quinto nível dessa classificação. Adicionalmente, foi avaliado o uso de medicamentos considerados potencialmente inadequados para idosos segundo os critérios de Beers, na sua última atualização, e segundo os critérios STOPP 18,14. Para esta análise, foram utilizados 25 dos 65 critérios STOPP e 20 dos 53 critérios de Beers 2012. A não utilização de todos os critérios disponíveis decorreu de falta de informação que permitisse a avaliação completa do critério, como, por exemplo, medicamentos e doenças não relatados pelos pacientes.

A variável resposta foi uso de medicamentos potencialmente inadequados nos últimos 15 dias. As variáveis explicativas foram: sexo, idade, escolaridade, percepção de saúde, restrição de atividades nos últimos 15 dias por motivo de saúde, capacidade funcional, história de internação hospitalar, número de doenças e polifarmácia.

A avaliação da capacidade funcional baseouse no relato dos sujeitos, considerando-se uma escala de 12 tipos de atividades, entre as atividades de vida diária (AVD) e atividades instrumentais de vida diária (AIVD). As AVD incluídas foram: banhar-se, vestir-se, comer sozinho, ir ao banheiro, caminhar de um cômodo a outro na casa e sair da cama em direção à cadeira. Já as AIVD foram: preparar ou cozinhar o alimento, usar o telefone, sair de casa e pegar um ônibus, tomar a medicação, manusear o dinheiro, fazer compras, arrumar a casa, lavar e passar. Com base no relato das atividades, foi adotada a seguinte classificação: (1) sem dificuldade; (2) com pouca dificuldade; (3) com muita dificuldade; (4) não capaz; e (5) não se aplica. Subsequentemente, essas categorias foram somadas. Para fins de análise estatística, a variável capacidade funcional foi dicotomizada em adequada e inadequada. Assim, os indivíduos que relataram alguma dificuldade em realizar seis ou mais atividades (categorias 2 e 3), ou aqueles que consideraram difíceis de executar pelo menos três atividades de 12 (categoria 4), foram considerados como tendo a capacidade funcional inadequada 25 .

A polifarmácia foi definida, neste estudo, como o uso simultâneo de cinco ou mais medicamentos nos últimos 15 dias. Esse critério foi ado- 
tado com base no estudo de Beloosesky et al. 26 . Foram estimadas as prevalências de uso de medicamentos potencialmente inadequados para a amostra total considerando ambos os critérios e seus respectivos intervalos de $95 \%$ de confiança (IC95\%).

As diferenças entre proporções foram testadas com o qui-quadrado de Pearson; as associações entre o uso de medicamentos potencialmente inadequados e as variáveis explicativas foram examinadas por meio do cálculo de razões de prevalência (RP) e os respectivos IC95\%. Para identificação das variáveis independentemente associadas ao uso inadequado de medicamentos, foi realizada análise multivariada, por meio da regressão de Poisson, com variância robusta. As variáveis que se associaram ao uso de medicamentos potencialmente inadequados com $\mathrm{p}$ $<0,20$ foram incluídas no modelo multivariado. No modelo final, foram mantidas as variáveis que se associaram à variável resposta com valor de $\mathrm{p}<0,05$.

Para todos os testes, considerou-se nível de 5\% de significância. O software utilizado para análises dos dados foi o Stata, versão 13.0 (StataCorp LP, College Station, Estados Unidos).

A pesquisa na qual o presente estudo se insere foi conduzida dentro dos padrões exigidos pela Declaração de Helsinque, tendo sido aprovada pelo Comitê de Ética em Pesquisa com Seres Humanos da Universidade Federal de Viçosa (protocolo no $027 / 2008$ ). Um termo de consentimento livre e esclarecido, assinado, foi obtido dos idosos ou respondentes próximos.

\section{Resultados}

Dos 621 idosos avaliados, 570 usaram pelo menos um medicamento nos últimos 15 dias, o que corresponde a $91,8 \%$. A idade média desses idosos foi de 71 anos (DP = 8,16), variando de 60 a 98 anos, dos quais 55,8\% eram mulheres. A média de medicamentos utilizados foi de 4,28 (DP = 2,72 ), variando de um a 18 medicamentos; a prevalência de polifarmácia foi de 39,3\%.

Foram utilizados 2.440 medicamentos; destes, conforme classificação ATC, 48,4\% eram do sistema cardiovascular, $15,2 \%$ eram do trato alimentar e metabolismo, 13,3\% eram do sistema nervoso, seguidos de $6 \%$ que pertenciam ao grupo sangue e órgãos formadores. Do total de medicamentos utilizados, foram classificados como potencialmente inadequados 367 (15\%), segundo os critérios de Beers, e 436 (17,9\%), segundo os critérios STOPP.

Na Tabela 1, encontram-se os dez medicamentos potencialmente inadequados mais frequentemente utilizados de acordo com os critérios de Beers. Observa-se que os dois medicamentos de maior frequência pertencem ao sistema cardiovascular, totalizando $30,5 \%$ do total de inadequados conforme esses critérios. Já a Tabela 2 apresenta os dez medicamentos po-

Tabela 1

Frequência dos dez medicamentos potencialmente inadequados mais utilizados segundo os critérios de Beers, de acordo com a classificação Anatomical Therapeutic Chemical (ATC) 3o nível. Viçosa, Minas Gerais, Brasil, 2009.

\begin{tabular}{lcc}
\hline Medicamento & Classificação ATC 3o nível & $\mathbf{n}(\%)$ \\
\hline Nifedipina de liberação imediata & $\begin{array}{c}\text { Bloqueadores seletivos dos canais de cálcio com } \\
\text { efeitos principalmente vascular }\end{array}$ & $62(16,9)$ \\
Metildopa & Agentes antiadrenérgicos de ação central & $50(13,6)$ \\
Clonazepam & Antiepilépticos & $45(12,3)$ \\
Dipirona em associação com anti- & Outros analgésicos e antipiréticos & $15(4,1)$ \\
histamínicos de primeira geração & Antidepressivos & $15(4,1)$ \\
Amitriptilina & Glicosídeos cardíacos & $15(4,1)$ \\
Digoxina & Antiarrítmicos & $13(3,5)$ \\
Amiodarona & Outros antibacterianos & $11(3,0)$ \\
Nitrofurantoína & Ansiolíticos & $11(3,0)$ \\
Diazepam & Anti-inflamatórios e antirreumáticos não esteroides \\
Diclofenaco & & $9(2,5)$ \\
Demais medicamentos & & $121(32,9)$ \\
Total & $367(100,0)$
\end{tabular}


Frequência dos dez medicamentos potencialmente inadequados mais utilizados segundo os critérios Screening Tool of Older Person's Prescriptions (STOPP), de acordo com a classificação Anatomical Therapeutic Chemical (ATC) 3o nível. Viçosa, Minas Gerais, Brasil, 2009.

\begin{tabular}{|c|c|c|}
\hline Medicamento & Classificação ATC 3o nível & n (\%) \\
\hline Omeprazol & $\begin{array}{c}\text { Medicamentos para úlcera péptica e doença do } \\
\text { refluxo gastresofágico }\end{array}$ & $87(20,0)$ \\
\hline Clonazepam & Antiepilépticos & $45(10,3)$ \\
\hline Glibenclamida & Medicamentos redutores da glicemia & $42(9,6)$ \\
\hline Ácido acetilsalicílico & Agentes antitrombóticos & $18(4,1)$ \\
\hline Digoxina & Glicosídeos cardíacos & $16(3,7)$ \\
\hline $\begin{array}{l}\text { Dipirona em associação com anti-histamínicos } \\
\text { de primeira geração }\end{array}$ & Outros analgésicos e antipiréticos & $14(3,2)$ \\
\hline Diclofenaco & Anti-inflamatórios e antirreumáticos não esteroides & $12(2,8)$ \\
\hline Diazepam & Ansiolíticos & $11(2,5)$ \\
\hline Amitriptilina & Antidepressivos & $10(2,3)$ \\
\hline Formoterol associado à budesonida * & Inalantes adrenérgicos & $10(2,3)$ \\
\hline Demais medicamentos & & $171(39,2)$ \\
\hline Total & & $436(100,0)$ \\
\hline
\end{tabular}

* A inadequação deveu-se ao uso desses fármacos em mais de uma apresentação.

tencialmente inadequados mais frequentemente utilizados segundo os critérios STOPP. Percebese que o primeiro e o terceiro medicamentos mais frequentes pertencem ao trato alimentar e metabolismo, correspondendo a $29,6 \%$ do total de medicamentos potencialmente inadequados considerando-se esses critérios.

Do total de 621 idosos estudados, 596 foram incluídos na avaliação da adequação pelos critérios de Beers e 618, pelos critérios STOPP. Esses números se devem fato de que três pacientes não puderam ser avaliados em ambos os critérios por não haver relato da dosagem do medicamento ácido acetilsalicílico. Além disso, 22 pacientes em uso de medicamentos inibidores seletivos da recaptação de serotonina não foram avaliados pelos critérios de Beers, pois o instrumento de coleta de dados utilizado não avaliou o risco de quedas nestes idosos. Os casos em que não houve relato de uso de medicamentos no período considerado foram classificados como "uso adequado" em ambos os critérios.

De acordo com os critérios de Beers, 261 idosos foram expostos a pelo menos um medicamento potencialmente inadequado, o que corresponde a uma prevalência de uso de medicamentos potencialmente inadequados de 43,8\% (IC95\%: $37,8 \%-47,8 \%)$. O número de medicamentos potencialmente inadequados utilizados variou de um a três. Já de acordo com os critérios STOPP, a prevalência de uso de medicamentos potencial- mente inadequados foi de 44,8\% (IC95\%: 40,9\%$48,8 \%$ ), que corresponde a 277 idosos. O número de medicamentos potencialmente inadequados utilizados variou de um a seis medicamentos. Um total de 185 (31\%) idosos utilizou pelo menos um medicamentos potencialmente inadequados por ambos os critérios simultaneamente. Por outro lado, ao se considerarem os idosos que utilizaram algum medicamentos potencialmente inadequados por qualquer um dos critérios, $57,9 \%$ responderam por esse grupo. Observa-se, na Tabela 3, a prevalência de uso de medicamentos potencialmente inadequados para cada um dos critérios, conforme as variáveis de interesse. Para ambos os critérios, as maiores prevalências de uso de medicamentos potencialmente inadequados foram observadas entre as mulheres, os idosos mais velhos, com pior avaliação da própria saúde, que relataram restrição de alguma atividade por motivo de saúde, com capacidade funcional inadequada, com história de hospitalização no último ano, com história de cinco ou mais doenças crônicas e que praticavam polifarmácia, em relação aos seus congêneres.

Na Tabela 4, ainda considerando-se ambos os critérios, observa-se associação significante na análise univariada entre uso de medicamentos potencialmente inadequados e sexo feminino, restrição de atividades nos últimos 15 dias por motivo de saúde, capacidade funcional inadequada, história de cinco ou mais doenças, his- 
Prevalência de uso de medicamentos potencialmente inadequados conforme os critérios Beers 18 e Screening Tool of Older Person's Prescriptions (STOPP), segundo características sociodemográficas, de condições de saúde, utilização de serviços de saúde e polifarmácia. Viçosa, Minas Gerais, Brasil, 2009.

\begin{tabular}{|c|c|c|c|c|}
\hline \multirow[t]{2}{*}{ Variáveis/Categorias } & \multicolumn{2}{|r|}{ Beers } & \multicolumn{2}{|c|}{ STOPP } \\
\hline & $\mathbf{n}$ & Prevalência (\%) & $\mathrm{n}$ & Prevalência (\%) \\
\hline \multicolumn{5}{|l|}{ Sexo } \\
\hline Masculino & 282 & 35,8 & 287 & 36,2 \\
\hline Feminino & 314 & 51,0 & 331 & 52,3 \\
\hline \multicolumn{5}{|l|}{ Faixa etária (anos) } \\
\hline $60-69$ & 297 & 41,8 & 309 & 41,4 \\
\hline $70-79$ & 209 & 43,5 & 215 & 45,6 \\
\hline 80 ou mais & 90 & 51,1 & 94 & 54,3 \\
\hline \multicolumn{5}{|l|}{ Escolaridade } \\
\hline Nunca estudou & 90 & 44,4 & 93 & 48,4 \\
\hline Até as séries iniciais do Ensino Fundamental & 383 & 45,2 & 395 & 45,6 \\
\hline Ensino Fundamental completo ou mais & 122 & 39,3 & 129 & 40,3 \\
\hline \multicolumn{5}{|l|}{ Percepção da saúde } \\
\hline Bom/Muito bom & 258 & 38,8 & 271 & 34,0 \\
\hline Regular & 278 & 46,4 & 287 & 52,6 \\
\hline Ruim/Muito ruim & 38 & 47,4 & 38 & 55,3 \\
\hline \multicolumn{5}{|l|}{ Restrição de atividades } \\
\hline Não & 502 & 41,4 & 523 & 42,3 \\
\hline Sim & 93 & 57,0 & 94 & 59,6 \\
\hline \multicolumn{5}{|l|}{ Capacidade funcional } \\
\hline Adequada & 495 & 40,6 & 516 & 42,1 \\
\hline Inadequada & 99 & 58,6 & 100 & 60,0 \\
\hline \multicolumn{5}{|l|}{ História de internação hospitalar } \\
\hline Não & 502 & 42,2 & 523 & 42,3 \\
\hline Sim & 93 & 52,7 & 94 & 59,6 \\
\hline \multicolumn{5}{|l|}{ Número de doenças } \\
\hline Até 4 & 369 & 34,7 & 383 & 30,0 \\
\hline 5 ou mais & 227 & 58,6 & 235 & 68,9 \\
\hline \multicolumn{5}{|l|}{ Polifarmácia } \\
\hline Não & 382 & 28,8 & 394 & 27,9 \\
\hline Sim & 214 & 70,6 & 224 & 74,6 \\
\hline
\end{tabular}

tória de internação hospitalar nos últimos doze meses e prática de polifarmácia. Adicionalmente para os critérios STOPP, observou-se associação significante entre uso de medicamentos potencialmente inadequados e idade igual ou superior a 80 anos, além de pior percepção da saúde. Na análise multivariada, de acordo com os critérios de Beers, o sexo feminino e a polifarmácia se mantiveram independentemente associados ao uso de medicamentos potencialmente inadequados. Já para os critérios STOPP, as variáveis independentemente associadas ao uso de medicamentos potencialmente inadequados foram o sexo feminino, a percepção de saúde regular e a polifarmácia.

\section{Discussão}

Por ser o grupo etário que cresce mais rapidamente no país, a intensa demanda dos idosos por medicamentos e serviços de saúde gera grande impacto nas políticas públicas no setor. Elevadas prevalências de uso de medicamentos, variando de $83,5 \%$ a $95,4 \%$, foram observadas em estudos brasileiros com idosos 21,26. Como se observa no presente estudo, uma porcentagem alta dos idosos $(91,8 \%)$ fez uso de, no mínimo, um fármaco nos 15 dias anteriores à pesquisa.

Com relação aos medicamentos utilizados pelos idosos, observa-se que o consumo maior foi para os fármacos que atuam no sistema car- 
Resultados das análises univariada e multivariada da associação entre características sociodemográficas, de condições de saúde, de utilização de serviços de saúde e o uso de medicamentos potencialmente inadequados. Viçosa, Minas Gerais, Brasil, 2009.

\begin{tabular}{|c|c|c|c|c|}
\hline \multirow[t]{2}{*}{ Variáveis/Categorias } & \multicolumn{2}{|c|}{ Beers } & \multicolumn{2}{|c|}{ STOPP } \\
\hline & RP bruta (IC95\%) & RP ajustada (IC95\%) & RP bruta (IC95\%) & RP ajustada (IC95\%) \\
\hline \multicolumn{5}{|l|}{ Sexo } \\
\hline Masculino & 1,00 & 1,00 & 1,00 & 1,00 \\
\hline Feminino & $1,42(1,18-1,72)$ & $1,22(1,01-1,47)$ & $1,44(1,20-1,74)$ & $1,27(1,06-1,51)$ \\
\hline \multicolumn{5}{|l|}{ Faixa etária (em anos) } \\
\hline $60-69$ & 1,00 & & 1,00 & 1,00 \\
\hline $70-79$ & $1,04(0,85-1,28)$ & - & $1,10(0,90-1,34)$ & $1,03(0,86-1,23)$ \\
\hline 80 ou mais & $1,22(0,96-1,56)$ & - & $1,31(1,04-1,65)$ & $0,96(0,79-1,18)$ \\
\hline \multicolumn{5}{|l|}{ Escolaridade } \\
\hline Nunca estudou & 1,00 & & 1,00 & \\
\hline Até as séries iniciais do Ensino & $1,02(0,79-1,31)$ & - & $0,94(0,74-1,19)$ & - \\
\hline \multicolumn{5}{|l|}{ Fundamental } \\
\hline Ensino Fundamental completo ou mais & $0,89(0,64-1,22)$ & - & $0,83(0,62-1,12)$ & - \\
\hline \multicolumn{5}{|l|}{ Percepção de saúde } \\
\hline Bom/Muito bom & 1,00 & 1,00 & 1,00 & 1,00 \\
\hline Regular & $1,20(0,98-1,46)$ & $1,02(0,84-1,24)$ & $1,55(1,27-1,89)$ & $1,32(1,09-1,60)$ \\
\hline Ruim/Muito ruim & $1,22(0,85-1,77)$ & $0,96(0,68-1,37)$ & $1,63(1,17-2,27)$ & $1,34(0,99-1,81)$ \\
\hline \multicolumn{5}{|l|}{ Restrição de atividades } \\
\hline Não & 1,00 & 1,00 & 1,00 & 1,00 \\
\hline Sim & $1,38(1,12-1,69)$ & $1,11(0,88-1,41)$ & $1,41(1,16-1,71)$ & $1,05(0,85-1,29)$ \\
\hline \multicolumn{5}{|l|}{ Capacidade funcional } \\
\hline Adequada & 1,00 & 1,00 & 1,00 & 1,00 \\
\hline Inadequada & $1,44(1,18-1,76)$ & $1,05(0,83-1,35)$ & $1,43(1,18-1,72)$ & $0,95(0,78-1,16)$ \\
\hline \multicolumn{5}{|l|}{ História de internação hospitalar } \\
\hline Não & 1,00 & 1,00 & 1,00 & 1,00 \\
\hline Sim & $1,25(1,00-1,55)$ & $1,01(0,80-1,26)$ & $1,50(1,17-1,72)$ & $1,12(0,94-1,35)$ \\
\hline \multicolumn{5}{|l|}{ Número de doenças } \\
\hline Até 4 doenças & 1,00 & & 1,00 & \\
\hline 5 ou mais doenças & $1,69(1,41-2,02)$ & - & $2,30(1,93-2,74)$ & - \\
\hline \multicolumn{5}{|l|}{ Polifarmácia } \\
\hline Não & 1,00 & 1,00 & 1,00 & 1,00 \\
\hline Sim & $2,45(2,05-2,93)$ & $2,30(1,89-281)$ & $2,67(2,24-3,19)$ & $2,44(2,02-2,95)$ \\
\hline
\end{tabular}

IC95\%: intervalo de 95\% de confiança; RP: razão de prevalência.

diovascular, seguido dos que agem no trato alimentar e metabolismo. Resultado similar foi encontrado em outros estudos brasileiros sobre o uso de medicamentos em idosos não institucionalizados 20,21,22,23.

As maiores prevalências de uso de medicamentos potencialmente inadequados segundo os critérios de Beers foram, respectivamente, para os fármacos nifedipina de liberação imediata e metildopa. O mesmo foi observado no estudo de Oliveira et al. ${ }^{24}$, realizado com pacientes da atenção primária no Brasil. Nifedipina está associada ao risco de hipotensão e de isquemia miocárdica, enquanto a metildopa pode aumentar o risco de hipotensão, bradicardia e efeitos adversos do sistema nevoso central 18.

O medicamento potencialmente inadequado de maior utilização segundo os critérios STOPP foi o omeprazol. Nascimento et al. 27 também identificaram este medicamento como potencialmente inadequado mais frequentemente prescrito entre idosos institucionalizados de um município mineiro. Fato semelhante foi observado nos estudos de Bradley et al. ${ }^{28}$, com pacientes da atenção primária na Irlanda do Norte, e de Regueiro et al. 29 , com pacientes ambulatoriais 
na Argentina, em que o exemplo mais comum de prescrição potencialmente inadequada foram os inibidores da bomba de prótons (IBP) na dosagem máxima terapêutica por mais de oito semanas. Gallagher et al. 14 recomenda a suspensão ou redução de doses dos IBP.

Dentre os dez medicamentos potencialmente inadequados mais frequentemente utilizados, seis foram coincidentes em ambas as listas: a digoxina, o clonazepam, o diazepam, a dipirona em associação com anti-histamínicos de primeira geração, a amitriptilina e o diclofenaco. Ambas as listas relatam que o uso da digoxina em dose superior a $125 \mu \mathrm{g} /$ dia não está associada a nenhum benefício adicional e pode aumentar o risco de toxicidade. Tais listas também apontam para o aumento do risco de sedação prolongada, comprometimento cognitivo, delírio, redução do equilíbrio, quedas e fraturas causados pelos benzodiazepínicos, como o clonazepam e o diazepam. Ademais, o uso de anti-histamínicos de primeira geração relaciona-se com o risco de sedação e efeitos adversos anticolinérgicos, como, por exemplo, confusão, boca seca e constipação. A amitriptilina, um antidepressivo tricíclico, também está associada com sedação e efeitos adversos anticolinérgicos, além de agravar o transtorno cognitivo em pacientes com demência e causar constipação grave quando em uso com um opiáceo ou um bloqueador dos canais de cálcio. Os anti-inflamatórios não esteroides (AINE), como o diclofenaco, podem prejudicar a função renal, exacerbar a hipertensão arterial e aumentar o risco de hemorragia gastrointestinal e úlcera péptica, exceto com uso simultâneo de antagonistas dos receptores H2, IBP ou misoprostol 14,18 .

Os demais medicamentos potencialmente inadequados mais frequentes dos critérios de Beers, nifedipina de liberação imediata, metildopa, nitrofurantoína e amiodarona, não são contemplados pelos critérios STOPP. O mesmo ocorre com os demais medicamentos potencialmente inadequados mais frequentes dos critérios STOPP que não são citados pelos critérios de Beers, como o uso de omeprazol na dosagem terapêutica máxima por mais de oito semanas, a glibenclamida com diabetes mellitus tipo 2, o ácido acetilsalicílico em dosagem superior a 150mg/ dia e o formoterol associado a budesonida, que se refere ao critério de uso de medicamentos de mesma classe.

A prevalência de uso de medicamentos potencialmente inadequados não variou expressivamente entre os critérios estudados, $43,8 \%$ para Beers e $44,8 \%$ para STOPP. As diferenças apresentadas entre alguns medicamentos potencialmente inadequados mais frequentes entre os critérios sugerem, por um lado, que a utilização destas duas listas de forma complementar pode levar a uma maior detecção do uso de medicamentos potencialmente inadequados em idosos no Brasil. Por outro lado, indica que a utilização dos critérios de Beers para inquéritos em saúde é uma alternativa satisfatória, em especial pelo fato de sua aplicação ser menos complexa (critérios mais explícitos) do que a requerida pelos critérios STOPP. Por último, aponta a necessidade de elaboração de critérios ou listas próprios para uso na realidade brasileira.

Apesar das críticas apontadas na Europa com relação aos critérios de Beers de 2003 15, pesquisas recentes têm demonstrado maior sensibilidade para os critérios de Beers 2012 18, quando comparados com os critérios STOPP e com os de critéios de Beers 2003 15. Blaco-Reina et al. 30, em estudo com idosos de uma comunidade na Espanha, verificaram medicamentos potencialmente inadequados presentes em 24,3\%, 35,4\% e $44 \%$ das prescrições, de acordo os critérios de Beers 2003 15, STOPP e critérios de Beers 2012 18, respectivamente. Já Baldoni et al. 23, em uma investigação com mil pacientes não institucionalizados cadastrados no Sistema Único de Saúde (SUS), observaram uso de medicamentos potencialmente inadequados por $48 \%$ deles segundo os critérios de Beers 2003 15, e por 59,2\% conforme critérios de Beers 2012 18. De acordo com os autores, essa diferença parece ser decorrente da inclusão, na lista atualizada de Beers, de medicamentos de alta prevalência de uso no Brasil, tais como clonazepam e diclofenaco 23 .

Outros estudos também demonstraram maior sensibilidade dos critérios de Beers 2003, quando comparados com os critérios STOPP. Chen et al. 31 avaliaram 460 idosos de quatro casas de repouso e verificaram que $32,7 \%$ do grupo utilizaram medicamentos potencialmente inadequados segundo os critérios de Beers 2003 15, contra $23,7 \%$ segundo os critérios STOPP 31 . No estudo de Vishwas et al. 32, com 540 idosos em ambiente hospitalar, os critérios de Beers 2003 também foram capazes de detectar mais casos de medicamentos potencialmente inadequados do que os critérios STOPP, respectivamente $24,6 \%$ e $13,3 \%$.

De forma diversa, vários estudos europeus demonstraram maior sensibilidade dos critérios STOPP, quando comparados com os critérios de Beers 2003 15. No estudo de Hamilton et al. 33, com idosos em ambiente hospitalar, foram identificados medicamentos potencialmente inadequados em $56,2 \%$ e em $28,8 \%$ de todos os pacientes de acordo, respectivamente, com critérios STOPP e de critérios de Beers. Ryan et al. 34, em um estudo com 1.329 pacientes idosos da 
atenção primária, demonstraram que os critérios STOPP detectaram $21,4 \%$ dos pacientes com prescrições potencialmente inadequadas, contra 18,3\% detectados pelos critérios de Beers. Já segundo Miquel et al. 35 , em pesquisa sobre a prescrição potencialmente inadequada em idosos em clínica geriátrica, atenção primária e casa de repouso, os critérios STOPP/START foram capazes de identificar mais casos do que Beers: $47 \%$ e $23 \%$, respectivamente. A prevalência de uso de medicamentos potencialmente inadequados em idosos também é bastante variável no Brasil. Utilizando-se os critérios de Beers 2003, as prevalências de uso de medicamentos potencialmente inadequados em idosos não institucionalizados em Ijuí, Rio Grande do Sul; Belo Horizonte e Diamantina, Minas Gerais; e Ribeirão Preto, São Paulo, foram $21,7 \%$, 33,5\%, $44,7 \%$ e $48 \%$, respectivamente 20,21,22,23. Com relação à lista de critérios de Beers 2012 18, observou-se prevalência de uso de medicamentos potencialmente inadequados em $59,2 \%$ de idosos não institucionalizados de Ribeirão Preto 23.

As diferenças na prevalência de uso de medicamentos potencialmente inadequados entre os estudos podem ser atribuídas a diversos fatores, tais como diferença entre os métodos de avaliação de medicamentos potencialmente inadequados utilizados e características da população-fonte, como idade, sexo, escolaridade, prevalência de uso de medicamentos e doenças prevalentes. Da mesma forma, deve-se considerar a disponibilidade dos fármacos nos diferentes países dos estudos, podendo ocorrer de um medicamento não ser comercializado ou se apresentar em uma dosagem diferente. Além disso, os hábitos de prescrição podem variar entre os profissionais de acordo com o perfil epidemiológico e pressões ideológicas e de mercado no que se refere à frequência de uso, duração do tratamento, dosagem, quantidade total de medicamento a ser utilizado, associações medicamentosas e indicação de fármacos que já estão em desuso em outros locais.

Observou-se forte associação positiva entre polifarmácia e o uso de medicamentos potencialmente inadequados, em ambos os critérios. Resultados semelhantes têm sido observados em investigações internacionais 30,31,32 e brasileiras 23,24,36. Como já relatado em estudos anteriores, a polifarmácia tem sido associada a resultados negativos em saúde, como, por exemplo, reações adversas, interações medicamentosas, baixa adesão e síndromes geriátricas 9,37,38. Corroborando esse quadro, a associação aqui observada evidencia um cenário de baixa qualidade da polifarmácia entre os idosos estudados, já que esta se constituiu em um fator de risco para o uso de medicamentos potencialmente inadequados. Entretanto, o manejo das diversas doenças crônicas nos idosos exige, na maioria das vezes, o uso de vários medicamentos, de modo que a polifarmácia nem sempre significa utilização incorreta dos medicamentos, justificando-se seu uso pela busca da melhora na qualidade de vida dos idosos com múltiplas doenças. Por isso, é preciso qualificar a polifarmácia para que não ocorra agravamento da saúde dos idosos.

A associação observada entre sexo feminino e uso de medicamentos potencialmente inadequados corrobora os resultados de outros estudos 23,36. O sexo feminino tem se mostrado um preditor robusto e consistente de uso de medicamentos em diferentes estudos. As hipóteses para esse cenário é que as mulheres são mais propensas a procurar os serviços de saúde e a receberem um diagnóstico. Em consequência, o número de medicamentos utilizados por elas tende a ser maior, bem como a exposição a substâncias cujo risco supera o benefício 2. Essa realidade evidencia a necessidade de aprimoramento da assistência farmacêutica voltada para esse subgrupo da população, mais vulnerável ao risco de iatrogenias.

A percepção de saúde regular se associou ao uso de medicamentos potencialmente inadequados segundo os critérios STOPP. É possível que a não observação de associação significante entre esse uso e pior autopercepção da saúde no modelo final seja atribuída ao tamanho amostral, insuficiente para demonstrar tal relação. De fato, a pior percepção da saúde também é um indicador evidenciado por diferentes estudos de maior utilização de serviços e tecnologias de saúde 39,40. Indivíduos idosos com pior percepção de saúde se percebem doentes com mais frequência e procuram mais os serviços de saúde, o que resulta em mais prescrições medicamentosas para este grupo. A maior preocupação do nosso achado resulta do fato de esse subgrupo, que já apresenta pior condição de saúde, estar exposto ao uso inadequado de medicamentos, o que pode contribuir para uma maior morbimortalidade desses idosos.

O presente estudo apresenta limitações. A primeira delas diz respeito ao fato de não ter sido possível utilizarem-se todos os critérios das listas na avaliação do uso de medicamentos potencialmente inadequados, tendo em vista que os dados são provenientes de um inquérito em saúde, com recortes na obtenção de informações sobre medicamentos e doenças. Esse fato não só restringe as comparações entre esta e as demais pesquisas, como também pode levar a uma subestimativa do desfecho estudado, sugerindo que o problema do uso inadequado de medicamentos entre 
idosos no Brasil seja de maior magnitude. Pode, ainda, interferir na identificação dos fatores associados ao uso de medicamentos potencialmente inadequados, no que diz respeito tanto à identificação dos fatores, quanto à magnitude da força das associações observadas. Nesse sentido, é preciso considerar a possibilidade de que alguns resultados seriam diferentes caso os critérios fossem aplicados na íntegra. Por outro lado, os determinantes do uso de medicamentos potencialmente inadequados identificados no presente estudo estão em consonância com a literatura internacional.

A segunda limitação está relacionada ao delineamento transversal, que não permite estabelecer a relação temporal entre polifarmácia e uso de medicamentos potencialmente inadequados. O uso de múltiplos medicamentos pode aumentar a chance de que algum destes seja considerado um medicamento potencialmente inadequado e predispor o paciente a eventos adversos. Por sua vez, a polifarmácia pode ser resultado da adição de um novo medicamento para tratar um evento adverso causado pelo uso de um medicamento inadequado.

\section{Resumen}

Se ha evaluado el uso de medicamentos potencialmente inadecuados entre ancianos de Viçosa, Minas Gerais, Brasil, bajo los criterios de Beers (2012) y los criterios STOPP, igual que los factores asociados a este uso. Se trata de un estudio transversal con 621 ancianos no institucionalizados, encuestados mediante una entrevista en su domicilio. Las variables explicativas fueron sexo, edad, escolaridad, percepción de la salud, restricción de actividades en los últimos 15 días, capacidad funcional, historia de ingreso hospitalario, número de enfermedades auto-referidas y polifarmacia. Se ha realizado un análisis de regresión de Poisson multivariado. Se ha observado una alta prevalencia de uso de medicamentos potencialmente inadecuados se-
Por último, cabe mencionar o fato de os dados analisados serem do ano de 2009. Algumas condutas terapêuticas podem ter se modificado nos últimos cinco anos, de forma que diferenças nas prevalências de uso de medicamentos potencialmente inadequados podem ser esperadas.

A despeito das limitações apontadas, os resultados deste e de outros estudos aqui abordados evidenciam que a prevalência de uso de medicamentos potencialmente inadequados é alta em todo o mundo. Particularmente no âmbito nacional, os resultados observados apontam a necessidade de estudos adicionais que investiguem criteriosamente os medicamentos potencialmente inadequados no grupo etário avaliado, com o intuito de se formularem listas desses medicamentos voltadas para a realidade da atenção primária brasileira, além de protocolos que subsidiem o aprimoramento da prescrição médica. Tais listas deverão ser atualizadas periodicamente para que possam se adaptar às mudanças de medicamentos comercializados e às evidências científicas. Em adição, devem ser incorporadas aos cenários de atenção à saúde do idoso, a fim de contribuir para o uso racional de medicamentos e para a qualidade de vida desse grupo etário. gún los criterios de Beers 2012 y STOPP, 43,8\% (IC95\%: 37,8\%-47,8\%) e 44,8\% (IC95\%: 40,9\%-48,8\%), respectivamente. El sexo femenino y la polifarmacia se mantuvieron asociados al uso de medicamentos potencialmente inadecuados de acuerdo con Beers. Ya para STOPP, las variables independientemente asociadas al uso de medicamentos potencialmente inadecuados fueron el sexo femenino, la percepción de salud regular y la polifarmacia. Son necesarios esfuerzos para reducir la práctica de la polifarmacia entre ancianos.

Utilización de Medicamentos; Polifarmacia; Salud del Anciano; Encuestas Epidemiológicas 


\section{Colaboradores}

G. A. Martins e A. Q. Ribeiro contribuiram na concepção, projeto, análise e interpretação dos dados; redação e revisão crítica relevante do conteúdo do artigo. F. A. Acurcio, S. C. C. Francischini e S. E. Priore colaboraram na revisão crítica relevante do conteúdo do artigo.

\section{Agradecimentos}

Ao CNPq e Capes pelo financiamento do projeto.

\section{Referências}

1. World Health Organization. The uses of epidemiology in the study of the elderly. Geneva: World Health Organization; 1984. (Technical Report Series, 706).

2. Instituto Brasileiro de Geografia e Estatística. Síntese de Indicadores Sociais: uma análise das condições de vida da população brasileira. ftp://ftp. ibge.gov.br/Indicadores_Sociais/Sintese_de_Indi cadores_Sociais_2013/SIS_2013.pdf (acessado em 20/Jun/2014).

3. Veras R. Envelhecimento populacional contemporâneo: demandas, desafios e inovações. Rev Saúde Pública 2009; 43:548-54.

4. Schramm JMA, Oliveira AF, Leite IC, Valente JG, Gadelha AMJ, Portela MC, et al. Transição epidemiológica e o estudo de carga de doença no Brasil. Ciênc Saúde Colet 2004; 9:897-908.

5. Desevo G, Klootwyk J. Pharmacologic issues in management of chronic disease. Prim Care Clin Office Pract 2012; 39:345-62.

6. Klotz U. Pharmacokinetics and drug metabolism in the elderly. Drug Metabolism Rev 2009; 41:6776.

7. Baldoni AO, Chequer FMD, Ferraz ERA, Oliveira DP, Pereira LRL, Dorta DJ. Elderly and drugs: risks and necessity of rational use. Braz J Pharm Sci 2010; 46:617-32.

8. Cooney D, Pascuzzi K. Polypharmacy in the elderly: focus on drug interactions and adherence in hypertension. Clin Geriatr Med 2009; 25:221-33.

9. Rozenfeld S, Fonseca MJM, Acurcio FA. Drug utilization and polypharmacy among the elderly: a survey in Rio de Janeiro City, Brazil. Rev Panam Salud Pública 2008; 23:34-43.
10. Medeiros EFF. Intervenção Interdisciplinar enquanto estratégia para o Uso Racional de Medicamentos em idosos. Ciênc Saúde Coletiva 2011; 16:3139-49.

11. Secoili SR. Polifarmácia: interações e reações adversas no uso de medicamentos por idosos. Rev Bras Enferm 2010; 63:136-40.

12. Buck MD, Atreja A, Brunker CP, Jain A, Suh TT, Palmer RM, et al. Potentially Inappropriate medication prescribing in outpatient practices: prevalence and patient characteristics based on electronic health records. Am J Geriatr Pharmacother 2009; 7:84-92.

13. Hajjar ER, Cafiero AC, Hanlon JT. Polypharmacy in elderly patients. Am J Geriatr Pharmacother 2007; 5:345-51.

14. Gallagher P, Ryan C, Byrne S, Kennedy J, O'Mahony D. STOPP/START (Screening Tool of Older Person's Prescriptions) and START (Screening Tool to Alert doctors to Right Treatment). Consensus validation. Int J Clinical Pharmacol Therapeutics 2008; 46:72-83.

15. Fick DM, Cooper JW, Wade WE, Waller JL, Maclean JR, Beers MH. Updating the Beers criteria for potentially inappropriate medication use in older adults. Arch Intern Med 2003; 163:2716-24.

16. Guaraldo L, Cano FG, Damasceno GS, Rozenfeld S. Inappropriate medication use among the elderly: a systematic review of administrative databases. BMC Geriatrics 2011; 11:79.

17. Beers MH, Ouslander JG, Rollingher I, Reuben DB, Brooks J, Beck JC. Explicit criteria for determining inappropriate medication use in nursing home residents: UCLA Division of Geriatric Medicine. Arch Intern Med 1991; 151:1825-32. 
18. The American Geriatrics Society 2012 Beers Criteria Update Expert Panel. American Geriatrics Society updated beers criteria for potentially inappropriate medication use in older adults. J Am Geriatr Soc 2012; 60:616-31.

19. Silveira ED, Muñoz GM, Montero EB, Sánchez CC, Gallagher PF, Cruz-Jentoft AJ. Prescripcion inapropiada de medicamentos em lós pacientes mayores: los critérios STOPP/START. Rev Esp Geriatr Gerontol 2009; 44: 273-9.

20. Pinto MCX, Ferré F, Pinheiro MLP. Potentially inappropriate medication use in a city of Southeast Brazil. Braz J Pharm Sci 2012; 48:79-86.

21. Araújo CMC, Magalhães SMS, Chaimowicz F. Uso de medicamentos inadequados e polifarmácia entre idosos do Programa Saúde da Família. Latin American Journal of Pharmacy 2010; 29:178-84.

22. Ribas C, Oliveira KS. Perfil dos medicamentos prescritos para idosos em uma Unidade Básica de Saúde do município de Ijuí-RS. Rev Bras Geriatr Gerontol 2014; 17:99-114.

23. Baldoni AO, Ayres LR, Martinez EZ, Dewulf NLS, Santos V, Pereira LRL. Factors associated with potentially inappropriate medications use by the elderly according to Beers criteria 2003 and 2012. Int J Clin Pharm 2014; 36:316-4.

24. Oliveira MG, Amorim WW, Jesus SR, Rodrigues VA. Factors associated with potentially inappropriate medication use by the elderly in the Brazilian primary care setting. Int J Clin Pharm 2012; 34: 626-32.

25. Nascimento CM, Ribeiro AQ, Cotta RMM, Acurcio FA, Peixoto SV, Priore SE, Franceschini SCC. Factors associated with functional ability in Brazilian elderly. Arch of Gerontol Geriatr 2012; 54:89-94

26. Beloosesky Y, Nenaydenko O, Nevo RFG, Adunsky A, Weiss A. Rates, variability, and associated factors of polypharmacy in nursing home patients. Clin Interv Aging 2013; 8:1585-90.

27. Acurcio FA, Silva AL, Ribeiro AQ, Rocha NP, Silveira $\mathrm{MR}$, Klein $\mathrm{CH}$, et al. Complexidade do regime terapêutico prescrito para idosos. Rev Assoc Med Bras 2009; 55:468-74.

28. Nascimento MMG, Pereira ML, Souza ACS, Ribeiro AQ, Dias-Junior CAC. Potential drug therapy problems on an elderly nursing home population. Latin American Journal of Pharmacy 2011; 30:1985-90.

29. Bradley MC, Fahey T, Cahir C, Bennett K, O'Reilly D, Parsons C, et al. Potentially inappropriate prescribing and cost outcomes for older people: a cross-sectional study using the Northern Ireland Enhanced Prescribing Database. Eur J Clin Pharmacol 2012; 68:1425-33.

30. Regueiro M, Mendy N, Cañás M, Farina HO, Nagel P. Uso de medicamentos en adultos mayores no institucionalizados. Rev Peru Med Exp Salud Pública $2011 ; 28: 643-47$
31. Blanco-Reina, Ariza-Zafra G, Ocana-Riola R, LeonOrtiz M. 2012 American Geriatrics Society Beers criteria: enhanced applicability for detecting potentially inappropriate medications in European older adults? A comparison with the Screening Tool of Older Person's Potentially Inappropriate Prescriptions. J Am Geriatr Soc 2014; 62:1217-23.

32. Chen LL, Tangiisuran B, Shafie AA, Hassali AA. Evaluation of potentially inappropriate medications among older residents of Malaysian nursing homes. Int J Clin Pharm 2012; 34:596-603.

33. Vishwas HN, Harugeri A, Parthasarathi G, Ramesh M. Potentially inappropriate medication use in Indian elderly: comparison of Beers' criteria and Screening Tool of Older Persons' potentially inappropriate Prescriptions. Geriatr Gerontol Int 2012; 12:506-14.

34. Hamilton H, Gallagher P, Ryan C, Byrne S, O'Mahony D. Potentially inappropriate medications defined by STOPP Criteria and the risk of adverse drug events in older hospitalized patients. Arch Intern Med 2011; 171:1013-9.

35. Ryan C, O'Mahony D, Kennedy J, Weedle P, Byrne S. Potentially inappropriate prescribing in an Irish elderly population in primary care. Br J Clin Pharmacol 2009; 68:936-47.

36. Miquel MDC, Cuervo MS, Silveira ED, Machuca IS, Gonzalez-Blazquez S, Errasquin BM, et al. Potentially inappropriate drug prescription in older subjects across health care settings. Eur Geriatr Med 2010; 1:9-14.

37. Faustino CG, Passarelli MCG, Filho WJ. Potentially inappropriate medications among elderly Brazilian outpatients. São Paulo Med J 2013; 131:19-26.

38. Shah BM, Hajjar ER. Polypharmacy, adverse drug reactions, and geriatric syndromes. Clin Geriatr Med 2012; 28:173-86.

39. Jyrkka J, Enlund H, Korhonen MJ, Sulkaval R, Hartikainen S. Patterns of drug use and factors associated with polypharmacy and excessive polypharmacy in elderly persons: results of the Kuopio 75+ study: a cross-sectional analysis. Drugs Aging 2009; 26:493-503.

40. Louvison MCP, Lebrão ML, Duarte YAD, Santos JLF, Malik AM, Almeida ES. Desigualdades no uso e acesso aos serviços de saúde entre idosos do município de São Paulo. Rev Saúde Pública 2008; 42:733-40.

41. Destro JR, Boing AF, d'Orsi E. Fatores associados à realização de consultas médicas por idosos no Sul do Brasil: estudo de base populacional. Rev Bras Epidemiol 2014; 17:692-704.

Recebido em 23/Ago/2014

Versão final reapresentada em 22/Abr/2015

Aprovado em 04/Mai/2015 